\title{
The interaction of $\mathrm{cD}$ galaxies with the surrounding ICM - The case for Centaurus
}

\author{
Renato A. Dupke ${ }^{1}$ and Joel N. Bregman ${ }^{1}$ \\ ${ }^{1}$ Department of Astronomy, University of Michigan, Ann Arbor, MI 48109, USA \\ email: rdupke@umich.edu
}

\begin{abstract}
The excellent spatial resolution of the Chandra satellite allowed us to see a wide variety of new types of substructures in the intergalactic gas in clusters of galaxies. The most promising scenarios to explain at least some of the phenomenology in the clusters centers involve processes that result from the interaction of the central active galaxy and its surroundings. Here, we present preliminary results of a Chandra velocity analysis of the Centaurus cluster. The results suggest that some of the core features may be interacting with pre-existent bulk gas flows in the cluster.
\end{abstract}

Centaurus (Abell 3526) is a Bautz-Morgan type I galaxy cluster, it has an optical redshift of 0.0104 and is one of the closest X-ray bright clusters. It has a cooling core with an equivalent "cooling flow" estimated mass rate of $<30-50$ solar masses per year (e.g. Allen \& Fabian 1994; Peres et al. 1998). Its X-ray emission is relatively smooth except in the central arcmin (NGC4696) where a significant amount of X-ray substructures is found (Sanders and Fabian 2002). The average temperature of the gas in the cluster is $\sim 3.5 \mathrm{keV}$. Early studies of galaxy velocities in the Centaurus cluster have shown the existence of bimodality in velocity space (Lucey et al. 1986; Stein et al. 1997). Two groups are clearly separated: Cen 30, centered on the cD galaxy (NGC 4696) and Cen 45 , associated with the galaxy NGC 4709 at $\sim 15^{\prime}$ from NGC 4696. They have average velocity differences of $\sim 1500 \mathrm{~km} \mathrm{~s}^{-1}$ and the general consensus is that Cen45 is being accreted by the main body of the Centaurus cluster (Cen30). Dupke \& Bregman (2001) performed the first X-ray velocity tomography study of this cluster with ASCA and discovered a velocity gradient along a direction roughly perpendicular to that of Cen45 (Figure 1 LEFT). ASCA coarse angular resolution and gain variations across the CCDs were the main uncertainties for velocity measurements. Multi-pointing observations with Chandra were able to determine velocities with a much higher precision as we show below.

Centaurus was observed by Chandra twice in April 2003, each observation had an exposure of $35 \mathrm{ksec}$. The center of the S3 CCD was aimed at the regions of maximal and minimal velocities as derived from the ASCA analysis. This was done to eliminate gain variations on these regions. We used Ciao 3.0.2 with CALDB 2.26 to screen the data. No flare-like periods were found and the resulting exposure time were 34.3 and $33.9 \mathrm{ksec}$. A gain map correction was applied together with PHA and pixel randomization. The ACIS particle background was cleaned as prescribed for VFAINT mode. Point sources were extracted and the background used in spectral fits was generated from blank-sky observations. Here we show the results of spectral fits with XSPEC V11.3 using an absorbed (wabs) mekal thermal emission model. Metal abundances were measured relative to the solar photospheric values. Spectral channels were grouped ( $>25 \mathrm{cnt} / \mathrm{chan}$ ) and energy ranges were restricted to $0.5-9.0 \mathrm{keV}$. The known degradation of ACIS low energy 

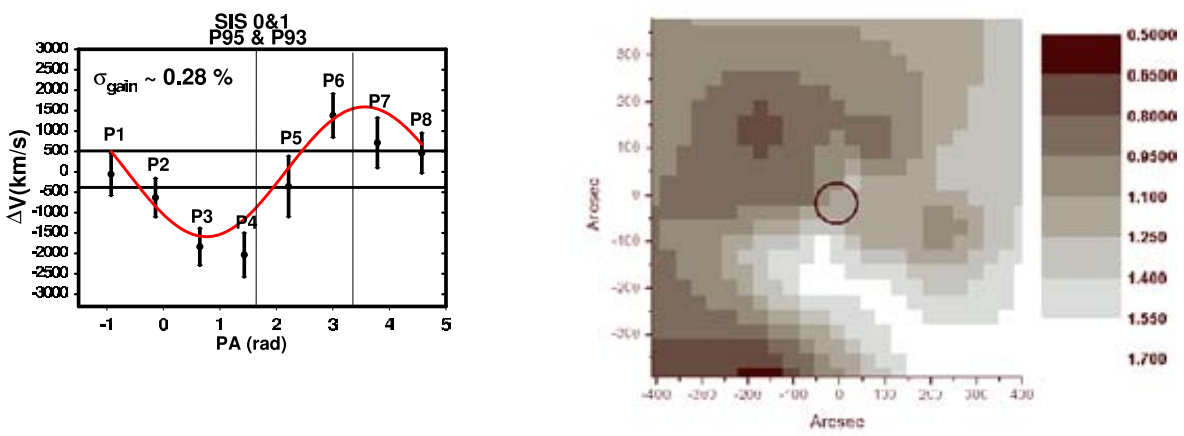

Figure 1. LEFT - Azimuthal velocity profile with respect to the center at 5 arcmin away from the center of the Centaurus cluster with ASCA North is towards P2. The horizontal lines show the $1 \sigma$ confidence limits for the core. The vertical lines show the direction towards Cen45. Errors are $1 \sigma$. RIGHT - Velocity map of the central regions of the Centaurus cluster with Chandra. The circle shows the central 1 arcmin. The scale shows redshifts in units of $10^{-2}$ so that 1.0 corresponds to $3000 \mathrm{~km} \mathrm{~s}^{-1}$. North is on top.

quantum efficiency (QE) was corrected automatically through the most recent versions of mkwarf and mkrmf routines (Chartas, G. 2004, private communication).

The results of spectral fittings for the centers of the CCDs are $2220 \pm 294 \mathrm{~km} \mathrm{~s}^{-1}$ (with corresponding gas temperatures $3.26 \pm 0.11 \mathrm{keV}$ and metal abundances $0.69 \pm 0.08$ Solar $\chi_{\nu}^{2}=1.314$ ) and $4977 \pm 297 \mathrm{~km} \mathrm{~s}^{-1}$ (with corresponding gas temperatures $3.43 \pm 0.17 \mathrm{keV}$ and metal abundances $0.68 \pm 0.11$ Solar $\left.-\chi_{\nu}^{2}=1.054\right)$. The velocity gradient measured with ASCA is confirmed with Chandra observations. We show a velocity map of the region analyzed by Chandra in Figure 1 RIGHT. We excluded the central arcmin since the amount of density and temperature substructures can easily cause spurious changes of the best fit velocities seen in the X-ray spectra. It can be seen from the velocity map that the direction of maximum velocity gradient is roughly perpendicular to that of the incoming sub-group Cen45 suggesting that the velocity gradient pre-existed the current merging event (e.g. Ricker 2000). The orientation of the velocity gradient axis is parallel to the X-ray arm seen in the core and suggests that the core substructures may be interacting with regions of high bulk velocities.

This research was partially supported by NASA Grants NAG5-13157 and NAG5-10806.

\section{References}

Allen, S. W., \& Fabian, A. C. 1994, MNRAS, 269, 409

Dupke, R. A., \& Bregman, J. N. 2001, ApJ, 562, 266

Lucey, J. R., Currie, M. J., \& Dickens, R. J. 1986, MNRAS, 221, 453

Peres, C. B., et al. 1998, MNRAS, 298, 416

Ricker, P. M. 1998, ApJ, 496, 670

Stein, P., Jerjen, H., \& Federspiel, M. 1997, A\&A, 327, 952 